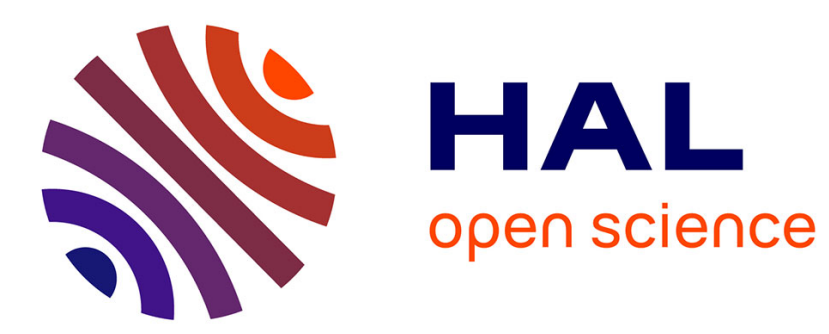

\title{
Exact simulation of nonlinear coagulation processes
}

Nicolas Fournier, Jean-Sébastien Giet

\section{To cite this version:}

Nicolas Fournier, Jean-Sébastien Giet. Exact simulation of nonlinear coagulation processes. Monte Carlo Methods and Applications, 2004, 10 (2), pp.95-108. hal-00143798

\section{HAL Id: hal-00143798 \\ https://hal.science/hal-00143798}

Submitted on 27 Apr 2007

HAL is a multi-disciplinary open access archive for the deposit and dissemination of scientific research documents, whether they are published or not. The documents may come from teaching and research institutions in France or abroad, or from public or private research centers.
L'archive ouverte pluridisciplinaire HAL, est destinée au dépôt et à la diffusion de documents scientifiques de niveau recherche, publiés ou non, émanant des établissements d'enseignement et de recherche français ou étrangers, des laboratoires publics ou privés. 


\title{
Exact simulation of nonlinear coagulation processes
}

\author{
Nicolas Fournier and Jean-Sébastien GieT \\ Institut Élie Cartan, \\ Campus Scientifique, BP 239 \\ 54506 Vandoeuvre-lès-Nancy Cedex, France \\ fournier@iecn.u-nancy.fr, giet@iecn.u-nancy.fr
}

\begin{abstract}
The Smoluchowski equation is a nonlinear integro-differential equation describing the evolution of the concentration $\mu_{t}(d x)$ of particles of mass in $(x, x+d x)$ in an infinite particle system where coalescence occurs. We introduce a class of algorithms, which allow, under some conditions, to simulate exactly a stochastic process $\left(X_{t}\right)_{t \geq 0}$, whose time marginals are given by $\left(x \mu_{t}(d x)\right)_{t \geq 0}$.
\end{abstract}

Key words : Coalescence, Simulation, Nonlinear jump processes.

$M S C 2000: 45 \mathrm{~K} 05,65 \mathrm{C} 05$.

\section{Introduction}

The Smoluchowski coagulation equation describes the evolution of an infinite particle system in which coalescence by pairs occurs. We assume that each particle is entirely characterized by its mass. We consider the concentration measure $\mu_{t}(d x)$ of particles having their masses in $(x, x+d x)$ at the instant $t \geq 0$. If one considers that two particles of mass $x$ and $y$ coalesce at rate $K(x, y)$, for some coagulation kernel $K$, then $\left(\mu_{t}\right)_{t \geq 0}$ satisfies the coagulation equation.

In [4], a pure jump nonlinear stochastic Markov process $\left(X_{t}\right)_{t \geq 0}$ has been associated with the coagulation equation, in the sense that for each $t \geq 0$, the law of $X_{t}$ is given by $x \mu_{t}(d x)$. This process may be seen as the evolution of the mass of a "typical" particle.

Our aim in this paper is to give a new existence proof of $\left(X_{t}\right)_{t \in[0, \infty)}$ (and thus of a solution to the Smoluchowski equation), by exhibiting an exact simulation algorithm. Our construction is thus explicit and direct. No approximation will be done. To our knowledge, the only existence proof not relying on successive approximations is due to Melzak, [11], and treats the case where $K$ is bounded 
(he also allows some fragmentation, and his proof relies on the use of series). Note that we do not obtain new existence results: our assumptions are, for example, stronger than those of Norris, [12].

We will treat in this paper both discrete case (i.e. when the particles have their mass in $\mathbb{N}^{*}$ ) and continuous case (i.e. when the particles have their mass in $(0, \infty))$. We will cover the case where $K(x, y)$ grows at most linearly at infinity. We do not allow fragmentation for simplicity, but the method could clearly be extended, at least in the discrete case and with a "reasonnable" fragmentation kernel. On the contrary, we do not deal with gelling kernels (for example $K(x, y) \geq(x y)^{\alpha}$ with $\left.\alpha>1 / 2\right)$, because our method would probably break down.

This study naturally leads to a new Monte-Carlo numerical scheme for the Smoluchowski equation, which we will compare to the stochastic finite particle method of Eibeck-Wagner [5].

The paper is organized as follows. In Section 2, we introduce the definitions of solutions to the coagulation equation, and of the nonlinear process $\left(X_{t}\right)_{t \geq 0}$. In Section 3, we describe a simulation algorithm for the discrete case. Section 4 is dedicated to the extension of the previous method to the continuous case. We finally present some numerical simulations in Section 5 .

The whole work is inspired by papers on the Boltzmann equation, especially those of Tanaka, [13], Graham-Méléard, [7], and Chauvin, [3].

\section{Definitions}

We first of all introduce some notations. For a subset $A$ of $(0, \infty)$, we denote by $\mathcal{M}_{f}^{+}(A)$ (resp. $\mathcal{P}(A)$ ) the set of nonnegative finite measures (resp. probability measures) on $A$. For a measure $\nu$ and a function $f$, we denote by $\langle\nu, f\rangle$ or $\langle\nu(d x), f(x)\rangle$ the integral $\int f d \nu$. Next we introduce some assumptions on the coagulation kernel $K$ and on the initial condition $\mu_{0}$, inspired by Norris, [12].

\section{Assumption $(L)$ :}

Let $E=\mathbb{N}^{*}$ or $E=(0, \infty)$. The initial condition $\mu_{0}$ belongs to $M_{f}^{+}(E)$ and satisfies $\left\langle\mu_{0}(d x), x\right\rangle=1$. The coagulation kernel $K$ is a measurable map on $E \times E$. There exists a continuous nonnegative function $\phi: E \mapsto[1, \infty)$ such that $x \mapsto \phi(x) / x$ is nonincreasing on $E$, and for all $x, y \in E$,

$$
0 \leq K(y, x)=K(x, y) \leq \phi(x) \phi(y)
$$

Furthermore, $\left\langle\mu_{0}(d x), x^{2}+\phi^{2}(x)\right\rangle<\infty$ and

$$
\begin{aligned}
\text { either } & x \mapsto \phi^{2}(x) / x \text { is nonincreasing on } E \\
\text { or } & \text { for all } x, y \in E, K(x, y) \leq \phi(x)+\phi(y)
\end{aligned}
$$

The advantage of such (complicated) assumptions is that it allows the coagulation kernel to explode at 0 in the continuous case. For example, the kernel $K(x, y)=\left(x^{1 / 3}+y^{1 / 3}\right)\left(x^{-1 / 3}+y^{-1 / 3}\right)$ fullfils $(L)$. 
Remark 2.1 If $E=\mathbb{N}^{*}$, one may assume (2.3) and choose $\phi(x)=A x$ for some constant $A \geq 1$ without loss of generality. Thus assumption $(L)$ reduces to $\left\langle\mu_{0}(d x), x\right\rangle=1,\left\langle\mu_{0}(d x), x^{2}\right\rangle<\infty$, and $0 \leq K(y, x)=K(x, y) \leq A(x+y)$.

We now give a definition of weak solutions to the Smoluchowski equation.

Definition 2.2 Assume $(L)$. A family $\left(\mu_{t}\right)_{t \geq 0}$ of measures of $M_{f}^{+}(E)$ solves the Smoluchowski equation $(S)$ if

(a) for all $t \geq 0,\left\langle\mu_{t}(d x), x\right\rangle \leq 1$ and $\sup _{[0, t]}\left\langle\mu_{s}(d x), \phi(x)\right\rangle<\infty$,

(b) for all test function $f \in C_{b}((0, \infty))$, all $t \geq 0$,

$$
\left\langle\mu_{t}, f\right\rangle=\left\langle\mu_{0}, f\right\rangle+\frac{1}{2} \int_{0}^{t}\left\langle\mu_{s}(d x) \mu_{s}(d y),[f(x+y)-f(x)-f(y)] K(x, y)\right\rangle d s
$$

Condition (a) ensures that under $(L)$ (see (2.1)), every term is finite in (2.4). The integral in the right hand side counts coagulation by pairs of particles of masses $x$ and $y$ at rate $K(x, y)$. We now define a related stochastic equation.

Definition 2.3 Assume $(L)$. A stochastic process $\left(X_{t}\right)_{t \geq 0}$ solves $(S D E)$ if there exists a filtered probability space $\left(\Omega, \mathcal{F},\left(\mathcal{F}_{t}\right)_{t \geq 0}, P\right)$ such that

(i) $\left(X_{t}\right)_{t \geq 0}$ is a càdlàg $E \cup\{\infty\}$-valued nondecreasing $\left(\mathcal{F}_{t}\right)_{t \geq 0}$-adpated process,

(ii) $X_{0}$ is $x \mu_{0}(d x)$-distributed,

(iii) there exists an $\left(\mathcal{F}_{t}\right)_{t \geq 0}$-adapted Poisson measure $N(d s, d y, d z)$ on $[0, \infty) \times$ $(E \cup\{\infty\}) \times \mathbb{R}_{+}$with intensity measure $d s Q_{s}(d y) d z$, where for each $s \geq 0, Q_{s}$ is the law of $X_{s}$, such that a.s., for all $t \geq 0$

$$
X_{t}=X_{0}+\int_{0}^{t} \int_{E \cup\{\infty\}} \int_{0}^{\infty} y \mathbb{1}_{\left\{z \leq \frac{K\left(X_{s-}, y\right)}{y}\right\}} \mathbb{1}_{\{y<\infty\}} N(d s, d y, d z)
$$

Since the integrand of the Poisson integral in (2.5) is nonnegative, the Poisson integral is well-defined as a Stieljes finite or infinite integral. The process $\left(X_{t}\right)_{t \geq 0}$ describes the evolution of the mass of a typical particle (see [4]). We collect in the following lemma some a priori estimates and the link between $(S D E)$ and $(S)$.

Lemma 2.4 Assume $(L)$. Consider a solution $\left(X_{t}\right)_{t \geq 0}$ to $(S D E)$. Then a.s., for all $t \geq 0, X_{t}<\infty$. For any $T<\infty$, $\sup _{[0, T]} E\left[X_{t}+\phi\left(X_{t}\right)\right]<\infty$. For each $t \geq 0$, denote by $Q_{t}=\mathcal{L}\left(X_{t}\right)$, and define the nonnegative finite measure $\mu_{t}$ on $E$ by $\mu_{t}(d x)=x^{-1} Q_{t}(d x)$. Then $\left(\mu_{t}\right)_{t \geq 0}$ solves $(S)$ and is conservative: for all $t \geq 0,\left\langle\mu_{t}(d x), x\right\rangle=1$.

Proof We give the main steps of the proof assuming (2.3), the case of (2.2) being similar.

Step 1 Note that thanks to $(L)$, there exists a constant $\alpha$ such that $\phi(x) \leq$ $\alpha x$ as soon as $x \geq 1$. Next, since $X$ is nondecreasing and thanks to $(L)$, $E\left[\phi\left(X_{t}\right) \mathbb{1}_{\left\{X_{t} \leq 1\right\}}\right] \leq E\left[\phi\left(X_{t}\right) / X_{t}\right] \leq E\left[\phi\left(X_{0}\right) / X_{0}\right]=\left\langle\mu_{0}, \phi\right\rangle<\infty$. 
Step 2 We first check that for all $T$, there exists a constant $C_{T}$ such that for all $t \leq T, E\left[X_{t}+\phi\left(X_{t}\right)\right] \leq C_{T}$. Thanks to step 1, it remains to prove that $E\left[X_{t}\right] \leq C_{T}$. For any $A \in[1, \infty)$, a simple computation shows that a.s.,

$$
X_{t} \wedge A \leq X_{0}+\int_{0}^{t} \int_{E} \int_{0}^{\infty}(y \wedge A) \mathbb{1}_{\left\{z \leq \frac{K\left(X_{s-}, y\right)}{y}\right\}} \mathbb{1}_{\left\{X_{s-} \leq A\right\}} N(d s, d y, d z)
$$

Using Step 1, $(L)$, and the expression of the intensity of $N$, we obtain

$$
E\left[X_{t} \wedge A\right] \leq a+a \int_{0}^{t}\left\{E\left[\phi\left(X_{s}\right) \mathbb{1}_{\left\{X_{s} \leq A\right\}}\right]+A E\left[\frac{\phi\left(X_{s}\right)}{X_{s}} \mathbb{1}_{\left\{X_{s} \geq A\right\}}\right]\right\} d s
$$

the constant $a$ being independent of $A$. Using Step 1, we deduce first that $E\left[\phi\left(X_{s}\right) \mathbb{1}_{\left\{X_{s} \leq A\right\}}\right] \leq a+a E\left[X_{s} \wedge A\right]$, and next that $A E\left[\mathbb{1}_{\left\{X_{s} \geq A\right\}} \phi\left(X_{s}\right) / X_{s}\right] \leq$ $a A P\left[X_{s} \geq A\right] \leq a E\left[X_{s} \wedge A\right]$. The Gronwall Lemma allows to conclude that for some constant $C_{T}$, all $t \leq T$, all $A \geq 1, E\left[X_{t} \wedge A\right] \leq C_{T}$. Making $A$ grow to infinity ends Step 2.

Step 3 We now prove that $\left(\mu_{t}\right)_{t \geq 0}$ solves $(S)$. It is clear from Step 2 that we may write, for any $t \geq 0$, any $g \in C_{b}^{1}((0, \infty))$,

$$
E\left[g\left(X_{t}\right)\right]=E\left[g\left(X_{0}\right)\right]+\int_{0}^{t} d s \int_{E} Q_{s}(d y) E\left[\frac{g\left(X_{s}+y\right)-g\left(X_{s}\right)}{y} K\left(X_{s}, y\right)\right]
$$

For any $f \in C_{c}^{1}((0, \infty))$, we may apply this formula to the function $g(x)=$ $f(x) / x$. Using the fact that $\mathcal{L}\left(X_{s}\right)=Q_{s}(d x)=x \mu_{s}(d x)$, we deduce that (2.4) holds with $f$. Formula (2.4) can be extended to any $f \in C_{b}((0, \infty))$, using Step 2 and the Lebesgue Theorem. Finally, $\left(\mu_{t}\right)_{t \geq 0}$ is of course conservative, since for each $t \geq 0, X_{t}<\infty$ a.s.

\section{The discrete case}

Our aim in this section is to build a solution to $(S D E)$ in the discrete case.

Assumption $(L D)$ : $(L)$ holds with $E=\mathbb{N}^{*}$. For $x, y \in \mathbb{N}^{*}$ we set

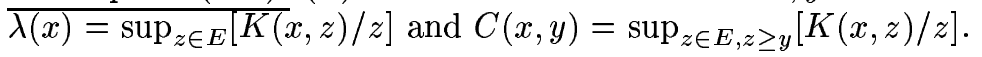

Note that $\lambda$ and $C$ are clearly well-defined, and that for each $x \in \mathbb{N}^{*}$, the map $y \mapsto C(x, y)$ is nonincreasing. Following carefully the proof below, one can check that:

Remark 3.1 If explicit computations of $\lambda$ and $C$ are impossible, one may replace them by any other functions $\tilde{\lambda}$ and $\tilde{C}$ such that for some constant a, for all $x, y \in \mathbb{N}^{*}, \lambda(x) \leq \tilde{\lambda}(x) \leq$ ax, and $C(x, y) \leq \tilde{C}(x, y)$. 


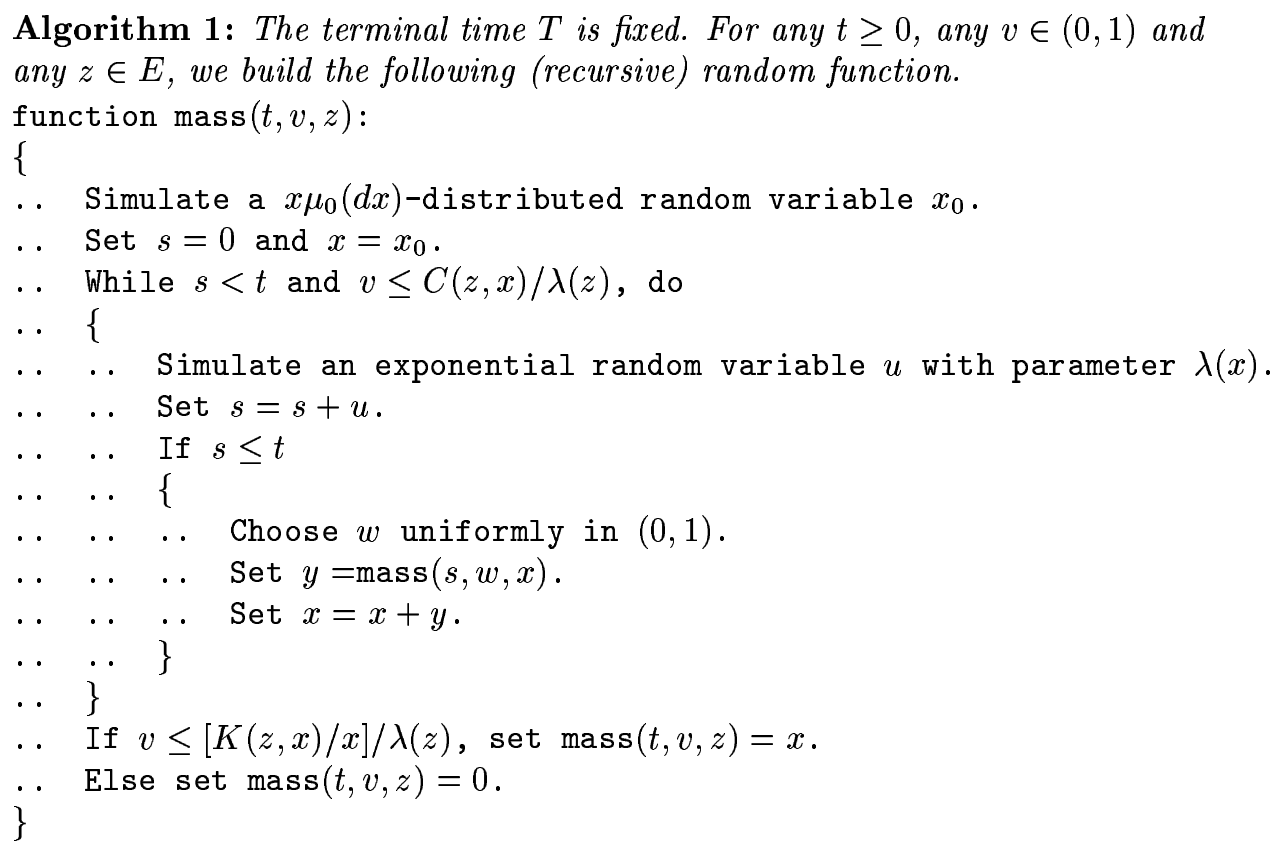

Then the way to build the process $X_{t}$ on the time interval $[0, T]$ is the following. Simulate a $x \mu_{0}(d x)$-distributed random variable $x_{0}$.

Set $s=0$ and $x=x_{0}$.

While $s<T$, do

\{

. Simulate an exponential random variable $u$ with parameter $\lambda(x)$.

. Set $X_{t}=x$ for all $t \in[s,(s+u) \wedge T)$.

. Set $s=s+u$.

. If $s \leq T$

.. \{

.. . Choose $w$ uniformly in $(0,1)$.

.. $\quad \ldots \quad$ Set $y=\operatorname{mass}(s, w, x)$.

... $\quad$ Set $x=x+y$.

$\cdots\}$

\}

The main idea of this algorithm consists in noting that the mass of a typical particle is obtained by adding, with well-chosen rates and acceptance-rejection procedures, the mass of other typical particles. The mass of these other typical particles will be obtained by adding, with well-chosen rates and acceptancerejection procedures, the mass of other typical particles, and so on... This explains why the algorithm we propose is recursive.

Proposition 3.2 Assume $(L D)$, and let $T<\infty$. Denote by $C_{T}$ the total number of times that Algorithm 1 calls the function mass. Then $E\left[C_{T}\right]<\infty$. In 
particular, Algorithm 1 ends a.s. Denote by $\left(X_{t}\right)_{t \in[0, T]}$ the obtained process. Then $\left(X_{t}\right)_{t \in[0, T]}$ satisfies $(S D E)$.

Proof Let $T$ be fixed. Recall that $E=\mathbb{N}^{*}$.

Step 0 First note that in the third line of function mass, the expression "While $s<t$ and $v \leq C(z, x) / \lambda(z)$ " can be replaced by "While $s<t$ ". This increases the time of computation, but does not change the result, since we anyway set $\operatorname{mass}(t, v, z)=0$ if $v \geq[K(z, x) / x] / \lambda(z)$. We handle the proof with this modification.

Step 1 For any $t \geq 0$, any $v \in(0,1)$, any $z \in E$, we denote by $P_{t, v, z}(d x, d c)$ the law of a couple of random variables $\left(X_{t, v, z}, C_{t, v, z}\right)$, where: $C_{t, v, z}$ is the (possibly infinite) number of times that mass $(t, v, z)$ calls the function mass, and $X_{t, v, z}$ is the result of the function mass $(t, v, z)$, with the convention that $X_{t, v, z}=0$ if $C_{t, v, z}=\infty$.

For each $t \geq 0$, we denote by $C_{t}$ the (possibly infinite) total number of times that algorithm 1 calls the function mass to obtain $X_{t}$. Then $C_{t}$ is a nondecreasing $\mathbb{N} \cup\{\infty\}$-valued process. We set $X_{t}=\infty$ if $C_{t}=\infty$.

Then, since $X_{t, v, z}$ is simulated essentially in the same way as $X_{t}$, in law,

$$
\left(X_{t, v, z}, C_{t, v, z}\right) \stackrel{(d)}{=}\left(X_{t} \mathbb{1}_{\left\{v \leq\left[K\left(z, X_{t}\right) / X_{t}\right] / \lambda(z)\right\}} \mathbb{1}_{\left\{X_{t}<\infty\right\}}, C_{t}\right)
$$

We finally denote, for each $t \in[0, T]$, by $Q_{t}$ the law of the $E \cup\{\infty\}$-valued random variable $X_{t}$.

Step 2 The process $\left(X_{t}\right)_{t \in[0, T]}$ is now well-defined as a càdlàg, $E \cup\{\infty\}$-valued, nondecreasing process, and $X_{0}$ has the law $x \mu_{0}(d x)$. We denote by $F$ the set $(E \cup\{0\}) \times(\mathbb{N} \cup\{\infty\})$. One may check that for each $t \in[0, T]$,

$$
\begin{aligned}
X_{t} & =X_{0}+\int_{0}^{t} \int_{0}^{1} \int_{F} x M(d s, d v, d(x, c)) \\
C_{t} & =\int_{0}^{t} \int_{0}^{1} \int_{F}(1+c) M(d s, d v, d(x, c))
\end{aligned}
$$

where $M(d s, d v, d(x, c))$ is a random integer-valued measure (see Jacod-Shiryaev, $[8])$ on $[0, T] \times(0,1) \times F$ with compensator $\lambda\left(X_{s-}\right) d s d v P_{s, v, X_{s-}}(d x, d c)$.

Step 3 We now show that $\left(X_{t}\right)_{t \in[0, T]}$ satisfies $(S D E)$. Using (3.1), one may rewrite (3.2) using a random integer-valued measure $O(d s, d v, d x)$ on $[0, T] \times$ $(0,1) \times(E \cup\{\infty\})$ with compensator $\lambda\left(X_{s-}\right) d s d v Q_{s}(d x)$, in the following way:

$$
X_{t}=X_{0}+\int_{0}^{t} \int_{0}^{1} \int_{E \cup\{\infty\}} x \mathbb{1}_{\left\{v \leq \frac{K\left(X_{s-}, x\right)}{\lambda\left(X_{s-}\right) x}\right\}} \mathbb{1}_{\{x<\infty\}} O(d s, d v, d x)
$$

which can finally be rewritten, using a Poisson measure $N(d s, d y, d u)$ on $[0, T] \times$ $(E \cup\{\infty\}) \times[0, \infty)$ with compensator $d s Q_{s}(d y) d u$, as

$$
X_{t}=X_{0}+\int_{0}^{t} \int_{E \cup\{\infty\}} \int_{0}^{\infty} y \mathbb{1}_{\left\{u \leq \frac{K\left(X_{s-}, y\right)}{y}\right\}} \mathbb{1}_{\{y<\infty\}} N(d s, d y, d u)
$$


Thus $\left(X_{t}\right)_{t \in[0, T]}$ satisfies $(S D E)$ in the sense of Definition 2.3. Thanks to Lemma 2.4, $(L D)$, and since $\lambda(x) \leq C(1+x)$ for some constant $C$, we deduce that

$$
a_{T}=\sup _{[0, T]} E\left[X_{t}+\lambda\left(X_{t}\right)\right]<\infty
$$

This implies in particular that $C_{T}<\infty$, and thus that Algorithm 1 ends a.s. Step 4 One finally needs to show that $E\left[C_{T}\right]<\infty$. First of all denote, for each $t \in[0, T]$, by $R_{t}$ the law of the $(\mathbb{N} \cup\{\infty\})$-valued random variable $C_{t}$. Using (3.1), one may rewrite (3.3) as

$$
C_{t}=\int_{0}^{t} \int_{\mathbb{N}}(1+c) \nu(d s, d c)
$$

where $\nu$ is a random integer-valued measure with compensator $\lambda\left(X_{s-}\right) d s R_{s}(d c)$. For each $A \in(0, \infty)$, we get $C_{t} \wedge A \leq \int_{0}^{t} \int_{\mathbb{N}}[(1+c) \wedge A] \nu(d s, d c)$. Taking expectation and using (3.6), we obtain

$$
E\left[C_{t} \wedge A\right] \leq \int_{0}^{t} d s E\left[\lambda\left(X_{s}\right)\right] \int_{\mathbb{N}}[(1+c) \wedge A] R_{s}(d c) \leq a_{T}+a_{T} \int_{0}^{t} E\left[C_{s} \wedge A\right] d s
$$

Using finally (3.6) and the Gronwall Lemma, we deduce that $E\left[C_{T} \wedge A\right] \leq B_{T}$ for some constant $B_{T}$ not depending on $A$. Making $A$ grow to infinity concludes the proof.

\section{The continuous case}

We now consider the possibly continuous case. The main difficulty comes from the fact that the function $\lambda(x)=\sup _{y \in E} K(x, y) / y$ can be infinite, because of small particles.

$$
\begin{aligned}
& \text { Assumption }(L C):(L) \text { holds with } E=(0, \infty) \text {. We set } a_{0}=\left\langle\mu_{0}, \phi\right\rangle \\
& \text { and, for each } x, y \in E, C(x, y)=\sup _{z \in E, z \geq y} K(x, z) / z .
\end{aligned}
$$

Note that $C$ is well-defined thanks to $(L)$.

Remark 4.1 If $C$ cannot be computed explicitely, one may replace it by any other function $\tilde{C}$ such that for all $x, y \in(0, \infty), \tilde{C}(x, y) \geq C(x, y)$.

The main idea is that problems due to small particles are problems that "decrease" when time increases, since all the particles have nondecreasing mass. The algorithm below consists in biasing the choice of initial particles.

Algorithm 2: The terminal time $T$ is fixed. For any $t \geq 0$, any $v \in(0,1)$ and any $z \in E$, we build the following (recursive) random function.

function $\operatorname{mass}(t, v, z)$ :

\{ 
. Simulate a $a_{0}^{-1} \phi(y) \mu_{0}(d y)$-distributed random variable $x_{0}$.

. Set $s=0$ and $x=x_{0}$.

.. While $s<t$ and $v \leq C(z, x) /\left[\phi(x) \phi\left(x_{0}\right) / x_{0}\right]$, do

.. \{

.. . Simulate an exponential random variable $u$ with rate $a_{0} \phi(x)$.

.. $\quad$ Set $s=s+u$.

.. If $s \leq t$

.. $\ldots\{$

.. . . . Choose $w$ uniformly in $[0,1]$.

.. . . . Set $y=\operatorname{mass}(s, w, x)$.

. $\quad \ldots \quad \ldots$ Set $x=x+y$.

$\cdots \quad \ldots\}$

$\ldots\}$

. If $v \leq[K(z, x) / x] /\left[\phi(z) \phi\left(x_{0}\right) / x_{0}\right]$, $\operatorname{set} \operatorname{mass}(t, v, z)=x$.

.. Else set $\operatorname{mass}(t, v, z)=0$.

\}

Then the way to build the process $X_{t}$ on the time interval $[0, T]$ is the following. Simulate a $y \mu_{0}(d y)$-distributed random variable $x_{0}$.

Set $s=0$ and $x=x_{0}$.

While $s<T$, do

\{

. Simulate an exponential random variable $u$ with parameter $a_{0} \phi(x)$.

. Set $X_{t}=x$ for all $t \in[s,(s+u) \wedge T)$.

. Set $s=s+u$.

.. If $s \leq T$

.. \{

.. . Choose $w$ uniformly in $[0,1]$.

. . . Set $y=\operatorname{mass}(s, w, x)$.

.. $\quad$. Set $x=x+y$.

$\ldots\}$

\}

Proposition 4.2 Assume $(L C)$, and let $T<\infty$. Denote by $C_{T}$ the total number of times that Algorithm 2 calls the function mass. Then $E\left[C_{T}\right]<\infty$. In particular, Algorithm 2 ends a.s. Denote by $\left(X_{t}\right)_{t \in[0, T]}$ the obtained process. Then $\left(X_{t}\right)_{t \in[0, T]}$ satisfies $(S D E)$.

Proof We just give the main ideas of the proof, since it is essentially the same as that of Proposition 4.2. Let $T \in(0, \infty)$ be fixed, and recall that $E=(0, \infty)$. We admit that $C_{T}<\infty$ a.s., and we show that the process $\left(X_{t}\right)_{t \geq 0}$ built by Algorithm 2 solves $(S D E)$. First $X$ is clearly $E$-valued, non-decreasing, and the law of $X_{0}$ is given by $x \mu_{0}(d x)$.

For $t \in[0, T], v \in(0,1)$, and $z \in E$, denote by $P_{t, v, z}\left(d x_{0}, d x\right)$ the law of a couple of random variables $\left(X_{t, v, z}^{0}, X_{t, v, z}\right)$, where $X_{t, v, z}^{0}$ is the initial value " $x_{0}$ " used to compute $\operatorname{mass}(t, v, z)$, and $X_{t, v, z}$ is the result of $\operatorname{mass}(t, v, z)$. 
For $t \in[0, T]$, we also denote by $P_{t}\left(d x_{0}, d x\right)$ the law of $\left(X_{0}, X_{t}\right)$, and by $Q_{t}$ the law of $X_{t}$.

On one hand, we may write $P_{t, v, z}\left(d x_{0}, d x\right)=a_{0}^{-1} \phi\left(x_{0}\right) \mu_{0}\left(d x_{0}\right) R_{t, v, z}\left(x_{0}, d x\right)$ and $P_{t}\left(d x_{0}, d x\right)=x_{0} \mu_{0}\left(d x_{0}\right) R_{t}\left(x_{0}, d x\right)$, and on the other hand, we have $Q_{t}(d x)=$ $\int_{x_{0} \in E} P_{t}\left(d x_{0}, d x\right)$. Then, since we simulate $X_{t, v, z}$ essentially in the same way as $X_{t}$, we deduce that for each $x_{0} \in E$,

$$
\mathbb{1}_{\{x>0\}} R_{t, v, z}\left(x_{0}, d x\right)=\mathbb{1}_{\left\{v \leq \frac{K(z, x) / x}{\phi(z) \phi\left(x_{0}\right) / x_{0}}\right\}} R_{t}\left(x_{0}, d x\right)
$$

The process $X$ can be shown to satisfy, for each $t \in[0, T]$,

$$
X_{t}=X_{0}+\int_{0}^{t} \int_{0}^{1} \int_{E} x M(d s, d v, d x)
$$

where $M$ is an integer-valued random measure on $[0, T] \times(0,1) \times E$ with compensator $a_{0} \phi\left(X_{s-}\right) d s d v \int_{x_{0} \in E} P_{s, v, X_{s-}}\left(d x_{0}, d x\right)$. Using (4.1), this can be rewritten as

$$
X_{t}=X_{0}+\int_{0}^{t} \int_{0}^{1} \int_{E \times E} x \mathbb{1}_{\left\{v \leq \frac{K\left(X_{s-}, x\right) / x}{\phi\left(X_{s-}\right) \phi\left(x_{0}\right) / x_{0}}\right\}} O\left(d s, d v, d\left(x, x_{0}\right)\right)
$$

the integer-valued random measure $O$ having the compensator $a_{0} \phi\left(X_{s-}\right) d s d v a_{0}^{-1} \phi\left(x_{0}\right) \mu_{0}\left(d x_{0}\right) R_{s}\left(x_{0}, d x\right)$. We deduce that

$$
X_{t}=X_{0}+\int_{0}^{t} \int_{0}^{\infty} \int_{E} x \mathbb{1}_{\left\{v \leq K\left(X_{s-}, x\right) / x\right\}} N(d s, d v, d x)
$$

the integer-valued random measure $N$ having the compensator $\nu(d s, d u, d x)=$ $\phi\left(X_{s-}\right) d s d u \int_{x_{0} \in E} \phi\left(x_{0}\right) \mu_{0}\left(d x_{0}\right) R_{s}\left(x_{0}, d x\right)\left[x_{0} / \phi\left(X_{s-}\right) \phi\left(x_{0}\right)\right]$.

But $\nu(d s, d u, d x)=d s d u \int_{x_{0} \in E} x_{0} \mu_{0}\left(d x_{0}\right) R_{s}\left(x_{0}, d x\right)$, and thus $\nu(d s, d u, d x)=$ $d s d u Q_{s}(d x)$. The proof is finished.

We conclude with some possible extensions of Algorithms 1 and 2.

Remark 4.3 1. Assume $(L)$ with $E=\mathbb{N}^{*}$. Then Algorithm 1 could be extended to simulate a E-valued process $\left(X_{t}\right)_{t \in[0, T]}$ whose law is of the form $x \mu_{t}(d x)$, where $\mu_{t}(d x)$ satisfies a discrete coagulation-fragmentation equation, with any reasonnable fragmentation kernel.

2. Algorithm 2 could not be extended to simulate a continuous coagulationfragmentation process: fragmentation would lead to create small particles, so that biasing the choice of initial particles would not suffice.

3. Assume $(L)$ with $E=\mathbb{N}^{*}$ or $E=(0, \infty)$, and with $K(x, y)=x y$ (and thus $\phi(x)=x)$. Then, using the fact that $K(x, y) / y$ does not depend on $y$, one may note that the acceptance-rejection procedures are inexistent in algorithms 1 and 2. One can thus use these algorithms to simulate $\left(X_{t} \wedge A\right)_{t \in[0, T]}$, for any $A \in(0, \infty)$, where for each $t \in[0, T]$, the law of $X_{t}$ is of the form $x \mu_{t}(d x)+$ 
$\left(1-\left\langle\mu_{t}(d x), x\right\rangle\right) \delta_{\infty}(d x),\left(\mu_{t}\right)_{t \in[0, T]}$ being a solution to the Flory coagulation equation: for any reasonable $f: E \mapsto \mathbb{R}$,

$$
\begin{array}{r}
\left\langle\mu_{t}, f\right\rangle=\left\langle\mu_{0}, f\right\rangle+\frac{1}{2} \int_{0}^{t}\left\langle\mu_{s}(d x) \otimes \mu_{s}(d y),[f(x+y)-f(x)-f(y)] x y\right\rangle d s \\
-\frac{1}{2} \int_{0}^{t}\left\langle\mu_{s}(d x), x f(x)\right\rangle\left(1-\left\langle\mu_{s}(d x), x\right\rangle\right) d s
\end{array}
$$

The principle is very simple: for example in the discrete case, use algorithm 1 with $\lambda(x)=C(x, y)=x$, and set $X_{t} \wedge A=A$ as soon as the computation of $X_{t}$ requires more than $A$ calls to the function mass.

5. It is well-known that for some coagulation kernels, solutions to $(S)$ cannot be conservative: for exemple, it is shown in [6] that if $K(x, y)=(x y)^{\alpha}$, with $\alpha \in(1 / 2,1]$, then there exists $t_{0}<\infty$ such that $\left\langle\mu_{t}(d x), x\right\rangle=1$ for $t<t_{0}$ but $\left\langle\mu_{t}(d x), x\right\rangle<1$ for all $t>t_{0}$. From the $(S D E)$ point of view, this means that for $t>t_{0}, P\left[X_{t}=\infty\right]>0$. In such a case, it seems clear that one may simulate, using Algorithm 1 or $2,\left(X_{t}\right)_{t \in[0, T]}$, for $T<t_{0}$. Under the assumption that for all $x \in E, \lim _{y \rightarrow \infty} K(x, y) / y=0$, it might be possible that Algorithms 1 and 2 allow to simulate $\left(X_{t} \wedge A\right)_{t \geq 0}$, for any $A<\infty$. This does not seem interesting from the numerical point of view, but it is an interesting mathematical question.

\section{About simulations}

It is known (see Norris, [12]) that equation $(S)$ has a unique solution $\left(\mu_{t}\right)_{t \geq 0}$ under $(L)$. Since we are able to simulate exactly the process $\left(X_{t}\right)_{t \geq 0}$, and since for each $t$, the law of $X_{t}$ is exactly $x \mu_{t}(d x)$, one may exploit algorithms 1 and 2 to approximate numerically $\left(\mu_{t}\right)_{t>0}$ : use $n$ times Algorithm 1 (or 2) to simulate independent $\left(X_{t}^{1}\right)_{t \in[0, T]}, \ldots,\left(X_{t}^{n}\right)_{t \in[0, T]}$, and approximate $\mu_{t}$ by $a_{t}^{n}=n^{-1} \sum_{i=1}^{n}\left(X_{t}^{i}\right)^{-1} \delta_{X_{t}^{i}}$.

Note that the convergence is straightforward from the standard law of large numbers, and that confidence intervalls may be given thanks to the (classical) central limit Theorem.

We would like to compare our algorithms with the so-called Mass Flow Algorithm introduced by Eibeck-Wagner, [5], following an idea of Babovski, [2]. This scheme, based on finite stochastic particle systems, is shown to be quite efficient in [5], at least compared to the famous Marcus-Lushnikov scheme ([10], [9]). We recall the MFA algorithm, exactly in the form presented in [5]. We use the notations of $(L)$.

Algorithm MFA: The terminal time $T$ and the number $n$ of particles are fixed.

Simulate i.i.d. $x \mu_{0}(d x)$-distributed random variables $x_{1}, \ldots, x_{n}$.

Set $t=0$.

While $t<T$

\{

. Compute $\tau=n^{-1} \sum_{i=1}^{n} \phi\left(x_{i}\right) \sum_{j=1}^{n} \phi\left(x_{j}\right) / x_{j}$. 
- Simulate an exponential random variable $s$ with rate $\tau$.

.. Set $t=t+s$.

.. Choose $i$ according to the law $\left\{\phi\left(x_{i}\right) / \sum_{l=1}^{n} \phi\left(x_{l}\right)\right\}_{i \in\{1, \ldots, n\}}$.

.. Choose $j$ according to the law $\left\{\left[\phi\left(x_{j}\right) / x_{j}\right] \sum_{l=1}^{n}\left[\phi\left(x_{l}\right) / x_{l}\right]\right\}_{j \in\{1, \ldots, n\}}$.

.. With probability $K\left(x_{i}, x_{j}\right) / \phi\left(x_{i}\right) \phi\left(x_{j}\right)$, set $x_{i}=x_{i}+x_{j}$.

\}

Approximate $\mu_{T}(d x)$ by $b_{T}^{n}=n^{-1} \sum_{i=1}^{n} x_{i}^{-1} \delta_{x_{i}}$.

Note that $\sum_{i=1}^{n} \phi\left(x_{i}\right)$ and $\sum_{j=1}^{n} \phi\left(x_{j}\right) / x_{j}$ can be computed easily at each step from the previous step. Note also that the simulation of $i$ (or $j$ ) is simplified if $\phi(x)$ is of the form $c$ (or $c x)$.

In the numerical results below, we will sometimes use the following advantage of our scheme: if one is only interested in particles smaller than $A$, for some $A$, then we can stop the simulation when $X$ becomes greater than $A$. This is of course not the case in the MFA scheme.

In all what follows, we consider the following mean errors (in percent)

$$
\begin{array}{r}
\zeta\left(a_{t}^{n}\right)=E\left[100 \frac{\left|\left\langle a_{t}^{n}(d x), x^{2}\right\rangle-\left\langle\mu_{t}(d x), x^{2}\right\rangle\right|}{\left\langle\mu_{t}(d x), x^{2}\right\rangle}\right] \\
\xi\left(a_{t}^{n}, z\right)=E\left[100 \frac{\left|\left\langle a_{t}^{n}(d x), \mathbb{1}_{\{x \leq z\}}\right\rangle-\left\langle\mu_{t}(d x), \mathbb{1}_{\{x \leq z\}}\right\rangle\right|}{\left\langle\mu_{t}(d x), \mathbb{1}_{\{x \leq z\}}\right\rangle}\right]
\end{array}
$$

and by $\zeta\left(b_{t}^{n}\right), \xi\left(b_{t}^{n}, z\right)$ the same quantities replacing $a$ by $b$. The expectations will be obtained by taking the mean over 10000 experiences.

In the 3 cases labelled (a), (b), (c) below, $\left(\mu_{t}\right)_{t \geq 0}$ is explicit, see Aldous [1].

(a) Consider the (discrete) case where $K(x, y)=1$ and $\mu_{0}=\delta_{1}$. For $t=2$, the simulation of $b_{2}^{1000}$ using MFA with $n=1000$ particles (and with $\phi \equiv 1$ ) requires $0.038 u$ (second). For the same time of computation, one may use algorithm 1 to compute $a_{2}^{7650}$. Stopping algorithm 1 as soon as $X$ becomes greater than 1 (resp. 5), one may simulate $\xi\left(a_{2}^{18000}, 1\right)\left(\right.$ resp. $\left.\xi\left(a_{2}^{8000}, 5\right)\right)$ in $0.038 u$. We obtain the following mean relative errors in percent:

\begin{tabular}{|r|c|c|c|}
\hline Relative error of & $\left\langle\mu_{2}(d x), x^{2}\right\rangle$ & $\left\langle\mu_{2}(d x), \mathbb{1}_{\{x \leq 1\}}\right\rangle$ & $\left\langle\mu_{2}(d x), \mathbb{1}_{\{x \leq 5\}}\right\rangle$ \\
\hline \hline MFA & $\zeta\left(b_{2}^{1000}\right) \sim 1.6$ & $\xi\left(b_{2}^{1000}, 1\right) \sim 3.6$ & $\xi\left(b_{2}^{1000}, 5\right) \sim 1.5$ \\
\hline Algo. 1 & $\zeta\left(a_{2}^{7650}\right) \sim 0.6$ & $\xi\left(a_{2}^{7650}, 1\right) \sim 1.5$ & $\xi\left(a_{2}^{7650}, 5\right) \sim 0.6$ \\
\hline Algo. 1 stopped at 5 & & $\xi\left(a_{2}^{8000}, 1\right) \sim 1.5$ & $\xi\left(a_{2}^{8000}, 5\right) \sim 0.6$ \\
\hline Algo. 1 stopped at 1 & & $\xi\left(a_{2}^{18000}, 1\right) \sim 1.0$ & \\
\hline
\end{tabular}

For $t=10$, the simulation of $b_{10}^{1000}$ (resp. $a_{10}^{800}$ ) requires $0.110 u$. Stopping algorithm 1 as soon as $X$ becomes greater than 1 (resp. 5), one may simulate 
$\xi\left(a_{10}^{11000}, 1\right)$ (resp. $\left.\xi\left(a_{10}^{1800}, 5\right)\right)$ in $0.110 u$. This gives, in percent:

\begin{tabular}{|r|c|c|c|}
\hline Relative error of & $\left\langle\mu_{10}(d x), x^{2}\right\rangle$ & $\left\langle\mu_{10}(d x), \mathbb{1}_{\{x \leq 1\}}\right\rangle$ & $\left\langle\mu_{10}(d x), \mathbb{1}_{\{x \leq 5\}}\right\rangle$ \\
\hline \hline MFA & $\zeta\left(b_{10}^{1000}\right) \sim 1.8$ & $\xi\left(b_{10}^{1000}, 1\right) \sim 12.9$ & $\xi\left(b_{10}^{1000}, 5\right) \sim 4.2$ \\
\hline Algo. 1 & $\zeta\left(a_{10}^{800}\right) \sim 2.0$ & $\xi\left(a_{10}^{800}, 1\right) \sim 16.7$ & $\xi\left(a_{10}^{800}, 5\right) \sim 5.8$ \\
\hline Algo. 1 stopped at 5 & & $\xi\left(a_{10}^{1800}, 1\right) \sim 10.9$ & $\xi\left(a_{10}^{1800}, 5\right) \sim 3.9$ \\
\hline Algo. 1 stopped at 1 & & $\xi\left(a_{10}^{11000}, 1\right) \sim 4.5$ & \\
\hline
\end{tabular}

(b) Consider the (continuous) case where $K(x, y)=1$, where $\mu_{0}(d x)=4 e^{-2 x} d x$. For $t=2$, the simulation of $b_{2}^{1000}$ using MFA with $n=1000$ particles (and with $\phi \equiv 1$ ) requires $0.085 u$ (second). For the same time of computation, one may use algorithm 2 (with $\phi \equiv 1$ ) to compute $a_{2}^{3450}$. Stopping algorithm 2 as soon as $X$ becomes greater than 1 (resp. 5), one may simulate $\xi\left(a_{2}^{10800}, 1\right)$ (resp. $\left.\xi\left(a_{2}^{3700}, 5\right)\right)$ in $0.085 u$. We obtain, in percent:

\begin{tabular}{|r|c|c|c|}
\hline Relative error of & $\left\langle\mu_{2}(d x), x^{2}\right\rangle$ & $\left\langle\mu_{2}(d x), \mathbb{1}_{\{x \leq 1\}}\right\rangle$ & $\left\langle\mu_{2}(d x), \mathbb{1}_{\{x \leq 5\}}\right\rangle$ \\
\hline \hline MFA & $\zeta\left(b_{2}^{1000}\right) \sim 1.8$ & $\xi\left(b_{2}^{1000}, 1\right) \sim 9.6$ & $\xi\left(b_{2}^{1000}, 5\right) \sim 4.7$ \\
\hline Algo. 2 & $\zeta\left(a_{2}^{3450}\right) \sim 0.9$ & $\xi\left(a_{2}^{3450}, 1\right) \sim 6.4$ & $\xi\left(a_{2}^{3450}, 5\right) \sim 3.0$ \\
\hline Algo. 2 stopped at 5 & & $\xi\left(a_{2}^{3700}, 1\right) \sim 6.4$ & $\xi\left(a_{2}^{3700}, 5\right) \sim 3.0$ \\
\hline Algo. 2 stopped at 1 & & $\xi\left(a_{2}^{10800}, 1\right) \sim 4.0$ & \\
\hline
\end{tabular}

(c) Finally consider the (discrete) case where $K(x, y)=x+y$ and $\mu_{0}=\delta_{1}$. For $t=2$, the simulation of $b_{2}^{1000}$ using MFA with $n=1000$ particles (and with $\phi(x)=2 x$ ) requires $2.58 u$ (second). For the same time of computation, one may use algorithm 1 to compute $a_{2}^{1800}$. Stopping algorithm 1 as soon as $X$ becomes greater than 1 (resp. 5), one may simulate $\xi\left(a_{2}^{80000}, 1\right)$ (resp. $\left.\xi\left(a_{2}^{23000}, 5\right)\right)$ in $0.038 u$. We obtain the following mean relative errors in percent:

\begin{tabular}{|r|c|c|c|}
\hline Relative error of & $\left\langle\mu_{2}(d x), x^{2}\right\rangle$ & $\left\langle\mu_{2}(d x), \mathbb{1}_{\{x \leq 1\}}\right\rangle$ & $\left\langle\mu_{2}(d x), \mathbb{1}_{\{x \leq 5\}}\right\rangle$ \\
\hline \hline MFA & $\zeta\left(b_{2}^{1000}\right) \sim 6.3$ & $\xi\left(b_{2}^{1000}, 1\right) \sim 9.5$ & $\xi\left(b_{2}^{1000}, 5\right) \sim 5.9$ \\
\hline Algo. 1 & $\zeta\left(a_{2}^{1800}\right) \sim 2.5$ & $\xi\left(a_{2}^{1800}, 1\right) \sim 7.6$ & $\xi\left(a_{2}^{1800}, 5\right) \sim 4.6$ \\
\hline Algo. 1 stopped at 5 & & $\xi\left(a_{2}^{23000}, 1\right) \sim 2.1$ & $\xi\left(a_{2}^{23000}, 5\right) \sim 1.3$ \\
\hline Algo. 1 stopped at 1 & & $\xi\left(a_{2}^{80000}, 1\right) \sim 1.1$ & \\
\hline
\end{tabular}

Of course, the above numerical results deal with cases where Algorithm 1 (or 2 ) is efficient. It seems numerically clear that MFA is better for large times.

The main interest of our algorithms is theoritical. They might however be used for precise small-time computations. They seem also quite efficient from the small particles point of view. An advantage of our method is that we can give (theoritical) confidence intervalls.

\section{References}

[1] D.J. Aldous, Deterministic and Stochastic Models for Coalescence (Aggregation, Coagulation): A Review of the Mean-Field Theory for Probabilists, Bernoulli, 5, no 1, 3-48, 1999. 
[2] H. Babovsky, On a Monte Carlo scheme for Smoluchowski's coagulation equation, Monte Carlo Methods Appl., Vol. 5, no 1, 1-18, 1999.

[3] B. Chauvin, Branching processes, trees and the Boltzmann equation, Math. Comput. Simulation, vol. 38, no 1-3, 135-141, 1995.

[4] M. Deaconu, N. Fournier and E. Tanré, A pure jump Markov process associated with Smoluchowski's coagulation equation, Ann. Probab., Vol. 30, no 4, 1763-1796, 2002.

[5] A. Eibeck and W. Wagner, Stochastic particle approximations for Smoluchowski's coagulation equation, Ann. Appl. Probab., Vol. 11, no 4, 1137$1165,2001$.

[6] M. Escobedo, S. Mischler, B. Perthame, Gelation in coagulation and fragmentation models, preprint 01-19 of the Ecole Normale Supérieure de Paris, 2001.

[7] C. Graham and S. Méléard, Stochastic particle approximations for generalized Boltzmann models and convergence estimates, Ann. Probab., vol. 25, no $1,115-132$, (1997).

[8] J. Jacod and A.N. Shiryaev, Limit Theorems for Stochastic Processes, Springer Verlag, (1987).

[9] A. Lushnikov, Some new aspects of coagulation theory, Izv. Akad. Nauk SSSR, Ser. Fiz. Atmosfer. I Okeana, vol. 14, no 10, 738-743, 1978.

[10] A. Marcus, Stochastic coalescence, Technometrics, vol. 10, 133-143, 1968.

[11] Z.A. Melzak, A scalar transport equation, Trans. Amer. Math. Soc., vol. $85,547-560,1957$.

[12] J.R. Norris, Smoluchowski's coagulation equation: uniqueness, nonuniqueness and hydrodynamic limit for the stochastic coalescent, Ann. Appl. Probab., vol. 9, no1, 78-109, 1999.

[13] H. Tanaka, Probabilistic treatment of the Boltzmann equation of Maxwellian molecules, Z. Wahrsch. Verw. Gebiete, vol 46, no 1, 67-105, 1978. 